\title{
Atherectomy Rotating Component Diameter
}

National Cancer Institute

\section{Source}

National Cancer Institute. Atherectomy Rotating Component Diameter. NCI Thesaurus.

Code C150244.

The diameter of the rotating component of an atherectomy device. 